forthcoming in International Journal of Industrial Organization

\title{
Non-comparative versus Comparative Advertising of Quality*
}

\author{
Winand Emons ${ }^{\dagger} \quad$ Claude Fluet ${ }^{\ddagger}$ \\ Universität Bern Université du Québec \\ CEPR, CIRPÉE à Montréal, CIRPÉE
}

Version: January 2012

\begin{abstract}
Two firms produce a good with a horizontal and a vertical characteristic called quality. The difference in the unobservable quality levels determines how the firms share the market. We consider two scenarios: In the first one, firms disclose quality; in the second one, they send costly signals thereof. Under non-comparative advertising a firm advertises its own quality, under comparative advertising a firm advertises the quality differential. In either scenario, under comparative advertising the firms never advertise together which they may do under non-comparative advertising. Moreover, under comparative advertising firms do not advertise when the informational value to consumers is small.
\end{abstract}

Keywords: quality, advertising, disclosure, signalling.

JEL: D82, L15, M37

${ }^{*}$ We thank Kong-Pin Chen, Uwe Dulleck, Marco Hahn, Martin Hellwig, Simon Lörtscher, Gerd Mühlheusser, Andras Niedermayer, Martin Peitz, Yossi Spiegel, and two referees for helpful comments. The usual disclaimer applies. Emons acknowledges the hospitality of the Department of Economics at Boston University.

${ }^{\dagger}$ Departement Volkswirtschaftslehre, Universität Bern, Schanzeneckstrasse 1, Postfach 8573, CH-3001 Bern, Switzerland, Phone: +41-31-6313922, Fax: +41-31-6313383, Email: winand.emons@vwi.unibe.ch.

${ }_{\ddagger}^{\ddagger}$ Université du Québec à Montréal, C.P. 8888, Suc. Centre-Ville, Montréal H3C 3P8, Canada, Phone: +1-514-9878386, Fax: +1-514-9878494, Email: fluet.claudedenys@uqam.ca. 


\section{Introduction}

Comparative advertising is any form of advertising that explicitly or by implication identifies a competitor or goods or services offered by a competitor. It was illegal in many European countries until the late 1990s. By contrast, in the US comparative advertising has been encouraged by the Federal Trade Commission since the 1970's. ${ }^{1}$ A 1997 EU directive changed the situation in Europe by legalizing comparative advertising subject to the restriction that it should not be misleading. ${ }^{2}$ European Competition Authorities now tend to agree with their American counterparts in that comparative advertising is an important tool in promoting competition. Comparative advertising increases consumers' information about alternative products. It allows consumers to evaluate the performance of particular products against other products, thus enabling more informed purchasing decisions.

Despite its importance there has been little economic analysis on comparative advertising. We will review this literature at the end of the introduction. In this paper we address the following questions. Does comparative advertising indeed generate more information for consumers than non-comparative advertising? Do firms advertise more once comparative advertising is allowed? Can the two advertising regimes be compared using welfare criteria?

To answer these questions we consider a product with a horizontal characteristic called design and a vertical characteristic called quality. Two firms produce different designs. Consumers do not observe quality before purchase. Prices cannot signal quality. ${ }^{3}$ The firms compete for customers by advertising their quality. We first analyze a pure disclosure framework. If a firm

\footnotetext{
${ }^{1}$ See, e.g., the "Statement of Policy Regarding Comparative Advertising", Washington 1979, www.ftc.gov/bcp/policystmt/ad-compare.htm.

${ }^{2}$ Directive 97/55/EC, see Barigozzi and Peitz (2006) for more details. European courts tend to follow a literal interpretation of quality claims, whereas U.S. courts ask whether consumers are actually misled.

${ }^{3}$ In section 2 we explain why prices are not used as a signalling device. Alternatively, we could assume that prices are regulated or that upstream manufacturers impose resale price maintenance on retailers.
} 
advertises, it discloses the truth; it cannot falsify as such. ${ }^{4}$ We compare two scenarios. In the first firms can only engage in non-comparative advertising, i.e., a firm may disclose its own quality but not the competitor's. In the second scenario, the firms can also engage in comparative advertising. In both scenarios advertising is costly and firms may, therefore, choose to remain silent.

In the non-comparative framework a firm advertises if its quality level is above a threshold. If the quality is below the threshold, a firm remains silent; the cost of sending the message is higher than the gain. When both firms' quality is below the threshold, neither advertises and they share the market equally. When only one firm's quality is above the threshold, the high quality firm advertises while the low quality one says nothing. The high quality firm then has more customers. When both firms have high quality, both advertise. This may be highly inefficient: if both firms have the same high quality, both advertise at a cost yet still share the market equally.

In the second scenario firms may also engage in comparative advertising, meaning that firms disclose the quality differential. When both advertising formats are possible, consumers interpret non-comparative advertisements as implying that the quality differential is actually small; had it been high, the firm would have disclosed the quality differential. This unraveling implies that firms do not use non-comparative advertising; they either send comparative messages or do not advertise at all. If the quality differential is small, neither firm advertises. If it is large, the high quality firm advertises while the low quality one is silent. In equilibrium the firms never advertise together.

Comparative advertising tends to perform better than non-comparative advertising. Firms do not advertise if the quality differential is small and the information is of little value to consumers. If, however, the quality differential is large, the high quality firm advertises while the low quality one remains

\footnotetext{
${ }^{4}$ Advertisements communicate, e.g., hard information about technical features. False advertising is deterred by the threat of lawsuits.
} 
silent. There is no duplication of advertisement expenditures. By contrast, when only non-comparative advertising is allowed, firms advertise their high quality independently of their rival's quality level. Both firms may then advertise even when the information is of little or no value to consumers.

Next we look at the case where advertisements cannot provide hard information. ${ }^{5}$ The firms now try to convince consumers of their quality (or the quality differential) by sending advertisements with expensive features such as highly paid celebrities expressing satisfaction with the product. By assuming that the costs depend on quality and satisfy the single-crossing property, we model persuasion as a signalling game. For instance, a celebrity may be reluctant to praise a product she experienced to be inferior. In equilibrium, if firms advertise, they spend money on expensive advertising to convince consumers of their quality. Consumers rationally infer the true quality from the advertisements. Otherwise, the equilibria have essentially the same structure as in the pure disclosure framework. In particular, in the signalling set-up the states of the world where firms advertise or are silent are exactly the same as under disclosure. The welfare comparison, however, is now somewhat less in favor of comparative advertising. Signalling costs may blur the picture, making the comparison more ambiguous.

Let us now review the literature. The marketing literature has discussed comparative advertising quite extensively; see Grewal et al. (1997) for a survey. There is, however, little economics literature on comparative advertising. Anderson and Renault (2009) consider comparative advertising with respect to horizontal characteristics. If qualities are sufficiently different, the low quality firm will disclose horizontal attributes of both products. The main difference with our approach is that advertising is costless.

Barigozzi, Garella, and Peitz (2009) consider an incumbent with known

\footnotetext{
${ }^{5}$ Our distinction between hard and soft information is standard. Real life situations often fall somewhat in between. In 2009 telecommunications companies in Canada engaged in legal fights over their advertising campaigns. Rogers Communications sued Bell Mobility for describing its network as "the best and most powerful". Earlier Rogers had been sued by Telus for claiming it had the "fastest and most reliable network". Arguably, the legal suits served as advertising instruments.
} 
quality facing an entrant with unknown quality. The entrant can choose generic advertising which is standard money burning to signal quality. Moreover, the entrant can choose comparative advertising which involves a comparison of the two firms' qualities; this involves the risk that the incumbent may sue. By resorting to comparative advertising, the entrant signals that he has a strong case. Comparative advertising can signal quality in those cases where generic advertising cannot. An important difference to our model is that only the entrant can choose to advertise.

Anderson et al. (2010 a,b) empirically study advertising in the US overthe-counter analgesics industry. Almost half the ad spending in their sample was on comparative advertisements; all firms had some comparative ads. Brands with better characteristics transmit more information. Comparative ads contain significantly more information than non-comparative selfpromoting advertisements. The evidence that all firms use comparative advertising is at odds with our finding that only one firm does so. One possible explanation is that in the analgesics market quality has multiple dimensions and firms claim superiority in dimensions where they perform better.

More generally, our analysis is related to the industrial organization literature on advertising as quality disclosure or quality signalling. Levin, Peck, and Ye (2009) analyze a duopoly where firms can disclose their own quality by presenting verifiable information. In Daughety and Reinganum (2008), a monopolist may choose between costly disclosure or signalling his quality through prices. These papers only allow for non-comparative advertising. Our analysis is also related to disclosure games with multiple interested parties sharing the same information, as in Milgrom and Roberts (1986b).

An important literature, going back to Milgrom and Roberts (1986a), analyzes quality signalling via prices or advertising as money burning. This literature has mainly dealt with the case of a monopolist, i.e., it has considered one-sender games. An exception is Daughety and Reinganum (2007) who consider signalling through prices in a duopoly. Two other exceptions, more closely related to the present analysis, are Hertzendorf and Overgaard 
(2001) and Fluet and Garella (2002). In these papers the duopolists know each other's quality. In the resulting equilibria, signalling is either through prices alone or through the price-advertising mix. In the present paper, signalling through prices is not feasible. Moreover, we focus on the case where both firms may jointly signal about the same variable, namely the quality differential.

The remainder of the paper is organized as follows. In the next section we describe the model and derive the equilibrium prices. Section 3 analyzes the pure disclosure and section 4 the signalling framework.

\section{The Model}

Consider two firms 1 and 2 which produce products having two characteristics. The first characteristic is horizontal; we call it design. Firm 1 produces design 1 and firm 2 produces design 2. For example, design could refer to the interface in an operating system (Mac OS X vs. the Microsoft Windows or Symbian vs. Android), the location of a vacation resort (mountains vs. seaside) or the place where a cigar is produced (Cuba or the Dominican Republic). The second characteristic is vertical and concerns the quality of a particular feature; we will refer to it as firm $i$ 's quality $q_{i} \in[0,1], i \in\{1,2\}$. Production costs are normalized to zero, i.e., they are independent of design and quality. Firm $i$ charges the price $p_{i}$.

There are three groups of consumers: a mass $\mathrm{M}$ of firm 1 loyal consumers, a mass $\mathrm{M}$ of firm 2 loyal consumers, and a mass 1 of quality-conscious consumers. All consumers wish to buy at most one unit of the product. Loyal consumers do not care about the feature's quality. The utility of a firm $i$ loyal consumer is $1-p_{i}$ if he buys from firm $i,-p_{j}$ if he buys from firm $j$, and 0 if he does not buy at all. It is straightforward to verify that in the absence of quality-conscious consumers the Bertrand equilibrium prices are equal to 1, yielding profit $M$ for each firm.

Let us now turn to quality-conscious customers who care about design 
and quality and thus have higher willingness-to-pay. A quality-conscious consumer's utility is given as

$$
U= \begin{cases}1-p_{1}+2 q_{1}-\theta, & \text { if he buys from firm } 1 \\ 1-p_{2}+2 q_{2}+\theta, & \text { if he buys from firm } 2 \\ 0, & \text { if he does not buy }\end{cases}
$$

where $\theta$ is uniform on $[-\hat{\theta}, \hat{\theta}], \hat{\theta}>1$. The parameter $\theta$ measures the intensity with which a consumer cares about design. If $\theta$ is close to zero, design is not of great importance for the consumer and he cares more about quality. By contrast, if $\theta$ is close to the boundaries of the support, the consumer is a design aficionado for whom quality is of minor importance. The larger $\hat{\theta}$, the more the average quality-conscious consumer cares about design. ${ }^{6}$

To derive demand of quality-conscious customers consider first the case where both prices are low enough for the market to be covered. Suppose for the time being that quality is observable before purchase. Which design a consumer chooses depends on prices, on his $\theta$, and on the difference in quality levels $x:=q_{2}-q_{1}$; the consumer $\theta$ buys from firm 2 rather than from firm 1 if $-p_{2}+2 q_{2}+\theta \geq-p_{1}+2 q_{1}-\theta$ or $\theta \geq-x+.5\left(p_{2}-p_{1}\right)$.

Firm 1's demand (market share) from quality-conscious consumers is

$$
\int_{-\hat{\theta}}^{-x+.5\left(p_{2}-p_{1}\right)} \frac{1}{2 \hat{\theta}} d \theta=\frac{1}{2}-\frac{x}{2 \hat{\theta}}-\frac{p_{2}-p_{1}}{4 \hat{\theta}} ;
$$

firm 2's demand is

$$
\int_{-x+.5\left(p_{2}-p_{1}\right)}^{\hat{\theta}} \frac{1}{2 \hat{\theta}} d \theta=\frac{1}{2}+\frac{x}{2 \hat{\theta}}-\frac{p_{1}-p_{2}}{4 \hat{\theta}} .
$$

Suppose both firms charge the same price. If $q_{1}=q_{2}$, equivalently $x=0$, both firms share the market of quality-conscious consumers; if $q_{1}<q_{2}$ or

\footnotetext{
${ }^{6}$ To rephrase quality-conscious customers in the standard, quality-augmented, Hotelling set-up: Consumers are uniformly distributed on $[-\hat{\theta}, \hat{\theta}]$. Firm 1 is located in $-\hat{\theta}$, firm 2 is located in $\hat{\theta}$. Consumer $\theta$ 's utility when he buys from firm $i$ is $U=1-p_{i}+2 q_{i}+\hat{\theta}-d_{i}$, where $d_{1}=|-\hat{\theta}-\theta|$ and $d_{2}=|\hat{\theta}-\theta|$ are the distances the consumer has to travel to firm $i, i \in\{1,2\}$.
} 
$x>0$, firm 2 has more than half of the market; if $q_{1}>q_{2}$ or $x<0$, firm 1 has more than half of the market. The marginal impact of $x$ on profits is $+(-) 1 / 2 \hat{\theta}$ : the less consumers care about design (the smaller $\hat{\theta}$ ), the higher the impact of the quality differential.

Next let us look at the case where firm 2 is a monopolist. Moreover, suppose $q_{2}=1$. We thus consider the best possibility for firm 2: it is a monopolist with the highest possible quality level; furthermore, consumers are aware of this quality so that the firm incurs no advertising expenditures. Consumer $\theta$ buys 2's product if $\theta+3-p_{2} \geq 0$. On the market segment of quality-conscious consumers firm 2 thus faces demand $.5-\left(p_{2}-3\right) / 2 \hat{\theta}$. Maximizing profits with respect to this group of consumers yields $p_{2}^{*}=\hat{\theta} / 2+$ $1.5>1$ and profits $\pi_{2}^{*}=\hat{\theta} / 8+.75+9 / 8 \hat{\theta} . \pi_{2}^{*}$ is the upper bound on profits that can be made with quality-conscious customers; firm 2's profit can only be lower if firm 1 competes or consumers do not observe the feature's quality so that the firm has to advertise at a cost.

Finally, take the two segments together. We may now state a preliminary result.

Lemma 1: If $M>\pi_{2}^{*}, p_{1}=p_{2}=1$ in equilibrium.

This result follows immediately. Each product is sold at a uniform price, i.e., firms cannot discriminate between quality-conscious and loyal consumers. If firm 2 charges $p_{2}=1$, it serves at least its loyal customers who generate profit $M$. If it charges $p_{2}>1$, it loses its loyal customers and serves, if at all, only quality-conscious customers. The maximum profit it can make on this market segment is $\pi_{2}^{*}$. If $M>\pi_{2}^{*}$, firm 2 prefers to serve both market segments, which it optimally does by charging $p_{2}=1$; by symmetry, this condition also ensures that firm 1 charges $p_{1}=1$ in equilibrium. We assume $M>\pi_{2}^{*}$ so that Lemma 1 holds.

As an example for our model think of cell phones. Customers tend to be loyal to different operating system. As the feature take the cell phone's cam- 
era. It is now virtually impossible to get a cell phone without a camera even though a lot of customers never use it. Yet some consumers, say, teenagers, use the camera and care about its quality. The cost of adding the camera is small and it allows firms to gain market share in this segment of the market. Similarly, a vacation resort may build additional sports facilities to attract quality-conscious customers; it refrains from increasing prices in order not to lose loyal customers.

It remains to be explained why a firm doesn't produce two versions of the product, one with the feature and one without. Here we assume that there are economies of scale in production, making one large production run cheaper than two small ones. Finally, firms add the feature to the product not to get higher prices but to gain market share. If, say, firm 2 adds the feature while 1 doesn't, 2 gains the market segment of quality-conscious customers; if 2 drops the feature while 1 hangs on to it, 2 loses its share of the qualityconscious consumers. Therefore, if the cost of the feature is low, it is indeed optimal for both firms to add the feature to the product.

Let us now turn to the information structure. Quality-conscious consumers know the designs but do not observe the quality levels: the products are experience goods. We assume that $\tilde{q}_{1}$ and $\tilde{q}_{2}$ are independent and uniformly distributed on the unit interval. Without any additional information consumers expect $E\left(\tilde{q}_{1}\right)=E\left(\tilde{q}_{2}\right)=.5$ and the firms share the market equally. Unless $q_{1}=q_{2}$, this allocation is inefficient. If consumers learn, say, $x>0$, consumers with $\theta \in[-x, 0]$ buy from firm 2 rather than firm 1 . When they buy from 1 , their surplus is $\int_{-x}^{0}\left(-\theta+2 q_{1}\right) / 2 \hat{\theta} d \theta$; buying from 2 generates the surplus $\int_{-x}^{0}\left(\theta+2\left(q_{1}+x\right)\right) / 2 \hat{\theta} d \theta$. Becoming informed about $x$ thus increases surplus by $x^{2} / 2 \hat{\theta}$. This expression also applies when $x<0$.

Informing consumers about quality therefore not only redistributes profits, but typically also enhances efficiency. To put it differently, advertising quality in our set-up is on the one hand combative, acting to redistribute consumers among firms; on the other hand it is informative, increasing consumer 
surplus. $^{7}$

In the sequel, we analyze how firms compete through advertising in order to increase their market share of quality-conscious consumers. The timing is as follows. In stage 0, each firm learns its own quality as well as the quality of its competitor; consumers learn their type. In stage 1, the firms simultaneously send messages about the qualities; this includes the possibility of saying nothing. In stage 2, consumers observe the messages, draw inferences, and make their purchasing decisions.

\section{Disclosure}

\subsection{Non-comparative advertising}

In this section each firm may inform consumers about its own quality but not about the quality of its competitor. If firm $i$ advertises, it sends the message $y_{i}=q_{i}$ at a fixed cost $\gamma$. Alternatively, the firm may remain silent which we denote by $y_{i}=\emptyset_{i}$; remaining silent involves no cost.

We confine our attention to monotonic strategies: If a firm discloses when the state is $\left(q_{1}, q_{2}\right)$, it also discloses in more favorable states. The state $\left(q_{1}^{\prime}, q_{2}^{\prime}\right)$ is (weakly) more favorable for firm $i$ than $\left(q_{1}, q_{2}\right)$ if $q_{i}^{\prime} \geq q_{i}$ and $q_{j}^{\prime} \leq$ $q_{j}, i, j \in\{1,2\}, i \neq j$. A more favorable state for firm $i$ obviously implies a larger quality differential $q_{i}-q_{j}$. The converse is, however, not true. For example, if both qualities go down, yet $q_{1}$ more so than $q_{2}$, the differential becomes more favorable for firm 2. Note that as an immediate implication of monotonicity, if, say, firm 1 does not advertise in some state, it also doesn't advertise in less favorable states.

In the first stage of the game firms choose simultaneously whether or not to advertise. In the second stage consumers observe the firms' actions and form beliefs $E\left(x \mid y_{1}, y_{2}\right)=E\left(q_{2} \mid y_{1}, y_{2}\right)-E\left(q_{1} \mid y_{1}, y_{2}\right)$. Consumers buy from the firm maximizing expected utility, i.e., consumers with $\theta<E\left(x \mid y_{1}, y_{2}\right)$

\footnotetext{
${ }^{7}$ For a survey of the different views on advertising see, e.g., Bagwell (2007).
} 
buy design 1 and the rest design 2. If firm 1 doesn't advertise, its profit (net of profits from loyal customers) is $.5-E\left(x \mid \emptyset_{1}, y_{2}\right) / 2 \hat{\theta}$; if it discloses $q_{1}$, profit is $.5-E\left(x \mid q_{1}, y_{2}\right) / 2 \hat{\theta}-\gamma$. Similarly, firm 2's profits are $.5+E\left(x \mid y_{1}, \emptyset_{2}\right) / 2 \hat{\theta}$ and $.5+E\left(x \mid y_{1}, q_{2}\right) / 2 \hat{\theta}-\gamma$. Firms choose their advertising strategy so as to maximize expected profits. We look for symmetric perfect Bayesian equilibria.

Let us now derive the firms' strategies. Fix $q_{2}$ and consider firm 1. Due to monotonicity, if firm 1 discloses at some quality level $q_{1}$, it will also disclose any quality above this level; if it is silent at $q_{1}$, it is also silent for any quality below this level. Firm 1 thus plays a threshold strategy of being silent if its quality is below some $q_{1}^{0}\left(q_{2}\right)$ and of disclosing if its quality is above the threshold.

Now consider the message $\left(\emptyset_{1}, q_{2}\right)$. Consumers know 2's quality; moreover, they know that $q_{1}$ is below $q_{1}^{0}\left(q_{2}\right)$. Therefore, consumers rationally expect $E\left(q_{1} \mid \emptyset_{1}, q_{2}\right)=q_{1}^{0}\left(q_{2}\right) / 2$. At the threshold, firm 1 is indifferent between disclosing and being silent. If it is silent, its profit is .5- $\left[q_{2}-q_{1}^{0}\left(q_{2}\right) / 2\right] / 2 \hat{\theta}$; if it discloses, its profit is $.5-\left[q_{2}-q_{1}^{0}\left(q_{2}\right)\right] / 2 \hat{\theta}-\gamma$. The firm is indifferent between advertising and silence for $q_{1}^{0}\left(q_{2}\right)=4 \hat{\theta} \gamma$. Note that firm 1's equilibrium strategy is independent of $q_{2}$. By symmetry, the same argument applies to firm 2. To sum up: ${ }^{8}$

Proposition 1: In the unique equilibrium, if $q_{i}<4 \hat{\theta} \gamma$, firm $i$ chooses $\emptyset_{i}$; consumers correctly expect $E\left(q_{i} \mid \emptyset_{i}\right)=2 \hat{\theta} \gamma$. If $q_{i} \geq 4 \hat{\theta} \gamma$, firm $i$ discloses by choosing $y_{i}=q_{i}, i \in\{1,2\}$.

The outcome is depicted in Figure 1. If both firms have quality levels below $4 \hat{\theta} \gamma$, neither advertises. Consumers rationally expect average quality $2 \hat{\theta} \gamma$ of each firm. The more consumers care about design or the higher the cost of advertising, the larger the non-advertising range. If one firm's quality

\footnotetext{
${ }^{8}$ When we call an equilibrium unique, we mean unique except for the behavior at possible thresholds where players are indifferent.
} 
level is below while the other firm's is above the threshold, the high quality one advertises while the low quality one doesn't. Consumers know the quality of the advertising firm and expect $2 \hat{\theta} \gamma$ of the non-advertising one. When both quality levels are above the threshold, both firms advertise. Consumers know both quality levels and thus the quality differential. The game has prisoners' dilemma features. If, say, $q_{1}=q_{2}>4 \hat{\theta} \gamma$, both firms advertise yet share the market equally. They spend resources on disclosing without raising consumer surplus.

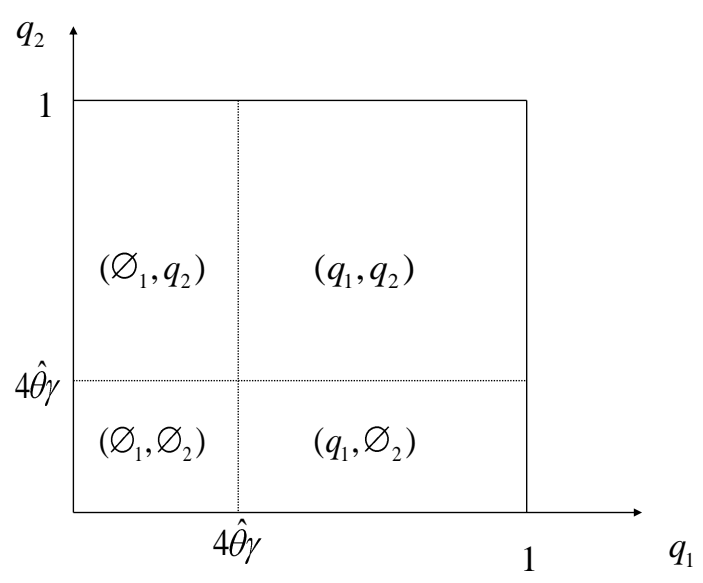

Figure 1: Non-comparative advertising

\subsection{Comparative advertising}

When comparative advertising is allowed, firms may disclose the difference in quality levels, the variable consumers are ultimately interested in; the cost of doing so is again $\gamma$. Firm $i$ may remain silent $\emptyset_{i}$, it may reveal its own quality $q_{i}$, or it may disclose both its quality and that of its competitor; the latter will be referred to as disclosure of the quality difference $x=q_{2}-q_{1} \cdot{ }^{9}$

\footnotetext{
${ }^{9}$ A firm may not engage im purely "negative advertising", i.e., disclose only the low quality of its competitor. Allowing this advertising format would not affect the results: our unraveling arguments also rule out negative advertising at equilibrium.
} 
The choice of the advertising format obviously has informational content. To see this, suppose firm 1 is silent while firm 2 discloses $q_{2}$. Consumers have to form beliefs about $q_{1}$. They use the information that firm 2 could have used comparative advertising at no additional cost but preferred not to do so. If $q_{1}$ is small and thus $x$ large, 2 will most likely use comparative advertising. If $q_{1}$ is large, firm 2 will prefer to disclose only $q_{2}$ and leave consumers in the dark about $q_{1}$. Accordingly, the non-comparative advertisement suggests that $q_{1}$ is large and thus $x$ small, making non-comparative advertising unattractive.

Before we can make these observations precise, we need to extend the concept of monotonic strategies: If a firm advertises in some state, it continues to do so in more favorable states. Moreover, if a firm uses comparative advertising in some state, it also does so in more favorable states. Monotonicity implies that when states become more favorable, a firm switches from no to non-comparative to comparative advertising or directly from no to comparative advertising. ${ }^{10}$

Lemma 2: If firms may engage in comparative as well as non-comparative advertising, they never use non-comparative advertising in equilibrium. Either they disclose $x$ or they remain silent.

Let us explain the main ideas. Consider first a pair of messages of the form $\left(q_{1}^{\prime}, x\right)$ where firm 1 advertises non-comparatively while firm 2 advertises comparatively. Such a pair cannot be part of an equilibrium: if firm 1 stops advertising, it doesn't change consumers' beliefs, yet saves the advertising cost. Suppose next that the pair $\left(\emptyset_{1}, q_{2}\right)$ is played at equilibrium. Let $S$ be the set of values for $q_{1}$ consistent with these messages. Denote by $\hat{q}_{1}$ the inference drawn by consumers, i.e., the expected value of firm 1's quality conditional on $S$. If this set is not a singleton, $\hat{q}_{1}>0$ and there are values $q_{1} \in S$ below $\hat{q}_{1}$. However, this yields a contradiction because firm 2 would

\footnotetext{
${ }^{10}$ When we use never we mean almost never, i.e., except possibly at isolated points in the state space.
} 
be better off revealing its rival's quality whenever $q_{1}<\hat{q}_{1}$. Hence $S$ must be a singleton, i.e., $S=\left\{\hat{q}_{1}\right\}$. Thus, the pair of messages $\left(\emptyset_{1}, q_{2}\right)$ can only arise when firm 1's quality is $\hat{q}_{1}$, that is, almost never; moreover consumers have exactly the same information and the outcome is the same as if firm 2 had used comparative advertising. Finally, a pair of messages $\left(q_{1}^{\prime}, q_{2}\right)$ also cannot be part of an equilibrium. If it were, monotonicity would imply that firm 2 also advertises at $q_{1} \leq q_{1}^{\prime}$. Suppose it sticks to non-comparative advertising. For $q_{1}$ sufficiently small, however, firm 1 will prefer not to advertise: it would rather be seen to have zero quality than pay $\gamma$ to be perceived as a low quality firm. Hence, for all values of $q_{1}$ sufficiently small, the pair $\left(\emptyset_{1}, q_{2}\right)$ would be played at equilibrium, contradicting the preceding argument that such a pair cannot be part of an equilibrium, except possibly at an isolated point. The proof is completed in the Appendix.

Given that firms only use comparative or no advertisements, the following result follows immediately. Note that since $\tilde{q}_{1}$ and $\tilde{q}_{2}$ are independent and uniform on the unit interval, $\tilde{x}$ has the unimodal density

$$
f(\tilde{x})= \begin{cases}1+x, & \text { if } x \in[-1,0) \\ 1-x, & \text { if } x \in[0,1]\end{cases}
$$

Proposition 2: In the unique equilibrium, if $x \in[-1,-2 \hat{\theta} \gamma]$, firm 1 discloses $x$ while firm 2 doesn't advertise. If $x \in(-2 \hat{\theta} \gamma, 2 \hat{\theta} \gamma)$, neither firm advertises. If $x \in[2 \hat{\theta} \gamma, 1]$, firm 2 discloses $x$ and firm 1 doesn't advertise. If neither firm advertises, consumers rationally expect a quality differential of zero.

Proof: Firms will never advertise together. If, say, firm 1 deviates to $\emptyset_{1}$ while firm 2 continues to disclose $x$, firm 1 doesn't change the consumers' decisions and saves the cost $\gamma$. Suppose consumers believe $E\left(x \mid \emptyset_{1}, \emptyset_{2}\right)=\hat{x}$ when neither firm advertises. Suppose firm 1 is silent. Firm 2 will disclose $x$ if $x-2 \hat{\theta} \gamma>\hat{x}$; otherwise, it is better off remaining silent. Likewise, firm 1 will disclose $x$ if $-x-2 \hat{\theta} \gamma>-\hat{x}$ and otherwise is silent. Therefore, consumers know that $x \in(\hat{x}-\hat{\theta} \gamma, \hat{x}+\hat{\theta} \gamma)$ when both firms do not advertise. Given that 
$f(x)$ is unimodal, $\hat{x}=E(x \mid x \in(\hat{x}-\hat{\theta} \gamma, \hat{x}+\hat{\theta} \gamma)]$ is possible only if $\hat{x}=0$.

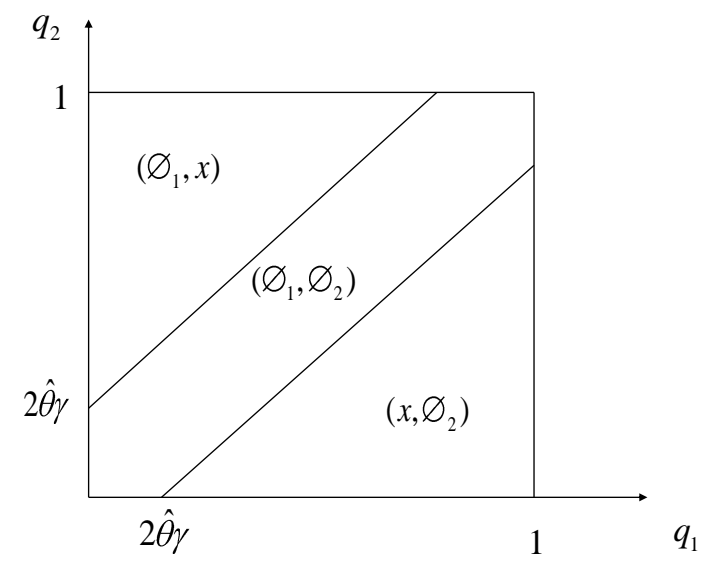

Figure 2: Comparative advertising

The outcome is depicted in Figure 2. Note that unlike the case of noncomparative advertising, the firms never advertise together. When the quality differential and thus the informational value to consumers is small, the firms do not advertise. Only when the differential is sufficiently large, the firm with the better quality advertises while the other firm remains silent. Unlike in the case of non-comparative advertising, a firm's strategy is not independent of the other firm's quality.

\subsection{Welfare}

To compare the welfare properties of our equilibria under non-comparative and comparative advertising consider Figure 3. Here we assume that $4 \hat{\theta} \gamma<1$, otherwise the firms never advertise when only non-comparative advertising is allowed.

Under non-comparative advertising no firm advertises in the areas $a$ and $b$; one firm advertises in the areas $c_{1}, c_{2}$ and $d$, providing imperfect information about the quality differential; in the areas $e$ and $f$ both firms advertise and provide perfect information. When comparative advertising is allowed, no 
firm advertises in $a, d$, and $f$; one firm advertises in the area $b, c_{1}, c_{2}$, and $e$, providing perfect information about the quality differential.

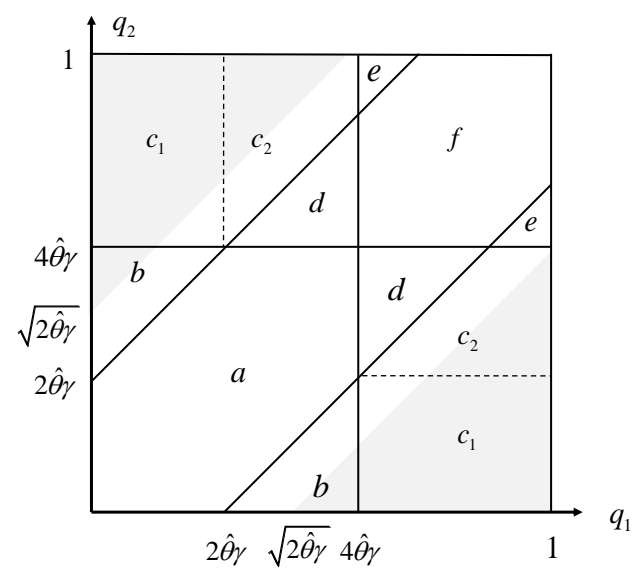

Figure 3: Welfare comparison

Recall that welfare increases by $x^{2} / 2 \hat{\theta}$ when consumers learn $x$. Advertising is socially desirable if the gain from informing consumers exceeds the cost. Advertising costs $\gamma$ when only one firm advertises. A necessary condition for advertising to be desirable is therefore $|x| \geq \sqrt{2 \hat{\theta} \gamma}$. Note that $2 \hat{\theta} \gamma<1$ implies $2 \hat{\theta} \gamma<\sqrt{2 \hat{\theta} \gamma}<1$. Advertising is thus inefficient in the non-shaded area of Figure 3.

In areas $a, d$, and $f$ advertising is inefficient: providing perfect information about $x$ is not worth the expenditure $\gamma$. In area $a$ firms don't advertise in both the non-comparative and the comparative scenario, thus welfare is the same. In areas $d$ and $f$ firms don't advertise under comparative advertising. Under non-comparative advertising one firm advertises in area $d$ and both firms advertise in area $f$. Therefore, comparative advertising performs better than non-comparative advertising in the areas $d$ and $f$.

In $c_{1}$ and $c_{2}$ one firm advertises under either regime. The non-comparative advertiser reveals only his own quality whereas the comparative advertiser discloses the quality differential. Thus, information is better under com- 
parative advertising. In $e$ the information is the same under both regimes. However, costs are duplicated under non-comparative advertising.

Finally, consider region $b$. When $\sqrt{2 \hat{\theta} \gamma}<4 \hat{\theta} \gamma$ as represented in the figure, region $b$ can be partitioned in two subregions: one where $x \leq \sqrt{2 \hat{\theta} \gamma}$ and one where $x>\sqrt{2 \hat{\theta} \gamma}$. In the first subregion disclosure by the comparative advertiser is not worth its cost. Hence, non-comparative advertising now does better because nobody advertises. In the second disclosure is efficient; under comparative advertising a firm discloses while under non-comparative advertising firms remain silent. When $\sqrt{2 \hat{\theta} \gamma}>4 \hat{\theta} \gamma$, advertising is not worth the cost in the whole of region $b$.

To sum up, allowing comparative advertising (weakly) improves welfare apart from region $b$ or a subarea thereof. Except for region $b$, comparative advertising has the following virtues. First, there is less advertising when the information is of little value to consumers (in $d$ and $f$ ). Second, ads are more informative: either consumers obtain more information for the same advertising expenditure (in $c_{1}$ and $c_{2}$ ) or the same information is conveyed at a lower cost because there is no duplication (in $e$ ). In region $b$ the welfare effects are ambiguous.

Finally, consider the case where $2 \hat{\theta} \gamma<1<4 \hat{\theta} \gamma$. Now firms do not advertise unless comparative advertising is allowed. In Figure 3 the only remaining regions are now $a$ and $b$. The welfare effects of comparative advertising are therefore ambiguous.

\section{Signalling}

Now consider the case where quality is unverifiable so that it cannot be simply disclosed. Firms will then attempt to persuade or convince consumers of their product's quality. We model this as a signalling game. Consumers are persuaded when they rationally infer quality from the costly signals sent by firms. As signals we take advertisements that make claims about the 
product's quality. Claiming high quality is easier when the product is indeed of high quality, i.e., the cost depends on the firm's actual quality or the quality differential. We focus on separating equilibria.

\subsection{Non-comparative advertising}

The cost of advertising is now given by $c(y, q)$ where $y$ is the message that the advertisement embodies. In addition to the many features it can incorporate, $y$ is taken to have "literal meaning" concerning the quality of the product. ${ }^{11}$ For example, in an ad featuring a SUV, the literal meaning is that the vehicle has extraordinary maneuverability even in the roughest and most exotic of terrains. The message is a claim or story conveyed in such a way that the literal meaning looks real. This is so even when the claim exceeds the range of feasible qualities and would, therefore, be perceived as somewhat fanciful. The costs of claims that look real depend on the product's actual quality. Therefore, under appropriate conditions, $y$ can play the role of a signal.

The function $c(y, q)$ reaches a strict global minimum at $y=q$ with respect to $y$ in which case $c(q, q)=\gamma$. Thus, the least costly claim is the one closest to the product's actual quality. The intuition is that for such a claim to look real requires no fabrication. Moreover, $c_{y}(y, q)=0$ has the unique solution $y=q$ and $c_{y q}(y, q)<0$. The last condition is the single-crossing property, i.e., the marginal cost of improving a story decreases with actual quality.

Denote consumers' inferences about quality by $\hat{q}_{i}, i \in\{1,2\}$. If firm 2 advertises, its payoff is $\pi_{2}=\left(.5-\hat{q}_{1} / 2 \hat{\theta}\right)+\hat{q}_{2} / 2 \hat{\theta}-c\left(y_{2}, q_{2}\right)$. We consider an equilibrium where $\hat{q}_{1}$ does not depend on the actions of firm 2. Accordingly, from the point of view of firm 2, the expression inside the parentheses is a constant, say, $k$. Dropping the subscript, firm 2's payoff is then $\pi=$ $k+\hat{q} / 2 \hat{\theta}-c(y, q)$. A similar expression holds for firm 1 .

Let $y=s(q)$ be the strategy played by a firm when it advertises. If the

\footnotetext{
${ }^{11}$ The expression is borrowed from Kartik's (2009) analysis of general one-sender communication games.
} 
strategy is separating, (i) it is monotonic and consumers infer $\hat{q}=s^{-1}(y)$ from the observation of $y$; (ii) it maximizes the firm's payoff given the inferences drawn by consumers. These conditions imply

$$
q=\arg \max _{\hat{q}} k+\hat{q} / 2 \hat{\theta}-\gamma-c(s(\hat{q}), q),
$$

i.e., the firm has no incentive to dissemble and the consumers' inferences are correct. The necessary first-order condition for (1) is

$$
1 / 2 \hat{\theta}-c_{y}(s(q), q) s^{\prime}(q)=0
$$

¿From our disclosure results we know that it is not worth advertising at a cost of $\gamma$ when the quality is less than $q^{0}=4 \gamma \hat{\theta}$. Accordingly, we take the solution to (1) with initial condition $s\left(q^{0}\right)=q^{0}$. The signalling strategies then satisfy the so-called Riley or least-cost signalling condition. It also follows that $s(q)$ solving (2) implies (1), i.e., the first-order condition is sufficient for a global maximum; see Mailath (1987) for the general signalling game with a continuum of types.

The single-crossing condition $c_{y q}<0$ implies $s^{\prime}(q)>0$ so that strategies are indeed monotonic. ${ }^{12}$ Note that $s^{\prime}(q)>0$ implies $c_{y}(s(q), q)>0$ over the range where the strategy is defined. Because $c(y, q)$ is minimized at $y=q$, the preceding inequality implies $s(q)>q$ for $q>q^{0}$. Thus, except at the threshold, advertising messages overstate the true quality.

Proposition 3: In a least-cost equilibrium, if $q_{i}<4 \hat{\theta} \gamma$, firm $i$ chooses $\emptyset_{i}$; consumers correctly expect $E\left(q_{i} \mid \emptyset_{i}\right)=2 \hat{\theta} \gamma$. If $q_{i} \geq 4 \hat{\theta} \gamma$, firm $i$ sends the message $y_{i}=s(q)>0$ solving (2) with $s(4 \gamma \hat{\theta})=4 \gamma \hat{\theta}$; consumers infer the true quality level $q_{i}, i \in\{1,2\}$.

\footnotetext{
${ }^{12}$ The necessary second-order condition is $-c_{y}(s(q), q) s^{\prime \prime}(q)-c_{y y}(s(q), q)\left(s^{\prime}(q)\right)^{2} \leq 0$. Differentiating (2) yields

$$
c_{y}(s(q), q) s^{\prime \prime}(q)+c_{y y}(s(q), q)\left(s^{\prime}(q)\right)^{2}+c_{y q}(s(q), q) s^{\prime}(q) \equiv 0,
$$

so that the second-order condition can be written as $c_{y q}(s(q), q) s^{\prime}(q) \leq 0$. Since $s^{\prime}(q)=0$ is inconsistent with $(2), s^{\prime}(q)>0$.
} 
In our signalling equilibrium the states of the world where firms advertise or are silent are exactly the same as in our pure disclosure framework; see Figure 1. Nevertheless, firms now spend additional resources to communicate their quality. The only way to credibly convey the true quality is to make costly claims whose literal meaning exaggerates the true quality. Since advertising truthfully is not incentive compatible, the equilibrium advertised qualities are distorted upward relative to the true quality levels. Consumers are rational, and hence they correctly infer the true quality from the advertising.

To illustrate, consider the quadratic cost function $c(y, q)=\gamma+.5(y-q)^{2}$. The equilibrium message is then the solution to

$$
y-\left[1-e^{-2 \hat{\theta}(y-4 \hat{\theta} \gamma)}\right] / 2 \hat{\theta}=q \text { with } y \geq q \geq 4 \hat{\theta} \gamma
$$

This strategy is shown in Figure 4. At equilibrium the advertising cost is increasing and concave in the product's true quality.

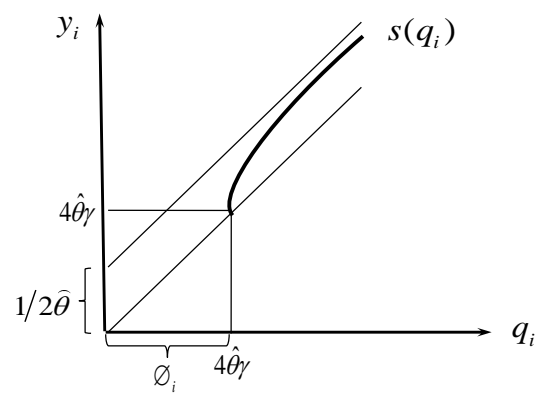

Figure 4: The signalling strategy with quadratic cost

\subsection{Comparative advertising}

Comparative advertisements mean that firms send messages $z_{i}, i \in\{1,2\}$ about the difference in quality levels $x=q_{2}-q_{1}$. The interpretation is again that $z_{i}$ has literal meaning and must look real. The cost of the message is $c\left(z_{i}, x\right)$. For parsimony we use the same notation as for non-comparative ads even though the function need not be the same. However, it satisfies the 
same basic conditions: $c_{z}(z, x)=0$ has the unique solution $z=x$ which minimizes $c(z, x)$ with respect to $z, c(x, x)=\gamma$, and $c_{z x}(z, x)<0$.

Firm $i$ may remain silent $\emptyset_{i}$, send a non-comparative signal $y_{i}$, or a comparative message $z_{i}$. As in the pure disclosure game the choice of the advertising format may have informational content. Yet, now advertising costs are not constant; they vary with the state of the world and the contents of the message. Moreover, the cost of a comparative message will in general differ from that of a non-comparative message. Therefore, we cannot show in general that firms will not use non-comparative ads when comparative advertising is allowed. Nevertheless, for the sake of comparison, we will exhibit equilibria where the firms only send comparative messages. In these equilibria, consumers may be described as expecting comparative ads. They hold beliefs that punish non-comparative advertisers. As a result, $y_{i}$ is never played at equilibrium. We make this precise in the proof of the next proposition.

When firm 1 advertises, its payoff is $\pi_{1}=.5-\hat{x} / 2 \hat{\theta}-c\left(z_{1}, x\right)$ where $\hat{x}$ is the consumers' inference about the quality differential. Suppose firm 2 remains silent and $\hat{x}$ depends only on the message $z_{1}$ sent by firm 1 . Applying the same argument as in the non-comparative section, the separating strategy $z_{1}=\sigma_{1}(x)$ must be a solution to the first-order condition

$$
-1 / 2 \hat{\theta}-c_{z}\left(\sigma_{1}(x), x\right) \sigma_{1}^{\prime}(x)=0
$$

We take the solution with initial condition $\sigma_{1}\left(x_{1}^{0}\right)=x_{1}^{0}$ where $x_{1}^{0}=-2 \hat{\theta} \gamma$, the same threshold as in the disclosure set-up. This ensures least-cost signalling. Similarly, when firm 2 advertises, its payoff is $\pi_{2}=.5+\hat{x} / 2 \hat{\theta}-c\left(z_{2}, x\right)$. If firm 1 is silent and $\hat{x}$ depends only on $z_{2}$, a separating strategy for firm 2 is $z_{2}=\sigma_{2}(x)$ solving the first-order condition

$$
1 / 2 \hat{\theta}-c_{z}\left(\sigma_{2}(x), x\right) \sigma_{2}^{\prime}(x)=0
$$

We take the solution with initial condition $\sigma_{2}\left(x_{2}^{0}\right)=x_{2}^{0}$ where $x_{2}^{0}=2 \hat{\theta} \gamma$. 
Again the same argument as for non-comparative ads show that $\sigma_{i}^{\prime}(x)>0$, $i \in\{1,2\}$. In equation (4) this implies $c_{z}\left(\sigma_{1}(x), x\right)<0$ over the range where the strategy is defined. In equation (5) it implies $c_{z}\left(\sigma_{2}(x), x\right)>0$ over the relevant range. Together with the initial conditions, the foregoing properties in turn imply that $\sigma_{1}(x)$ is defined for $x \leq x_{1}^{0}$ with $\sigma_{1}(x)<x$ when the differential is below the threshold; that $\sigma_{2}(x)$ is defined for $x \geq x_{2}^{0}$ with $\sigma_{2}(x)>x$ when the differential is above the threshold. Thus, the literal meaning of firm 2's message is to overstate the actual quality differential; that of firm 1 to understate it, equivalently to overstate the reverse differential $q_{1}-q_{2}$.

These separating strategies are derived following the approach of onesender signalling games; that is, each firm's strategy is obtained under the assumption that the other firm remained silent. In our set-up, however, both players share the same information and can communicate about the same variable, i.e., we have a two-sender game. This raises the possibility that both players could simultaneously advertise comparatively and send conflicting signals. One firm's signal could then "jam" the other firm's signal, see Kim (2003). Compared to the usual conditions of one-sender games, additional conditions are needed to preclude such a possibility. The following no-jamming conditions are sufficient for our purpose: The advertising cost function and the parameter $\hat{\theta}$ satisfy $c_{x x}(z, x) \geq 0, c_{x}(2 \hat{\theta} \gamma,-2 \hat{\theta} \gamma) \leq-1 / 4 \hat{\theta}$, $c_{x}(-2 \hat{\theta} \gamma, 2 \hat{\theta} \gamma) \geq 1 / 4 \hat{\theta}$. These conditions ensure that the difference between the literal meaning of a message and the true quality differential has a sufficiently large effect on advertising costs.

Proposition 4: The following strategies and beliefs constitute an equilibrium. If $x \in[-1,-2 \hat{\theta} \gamma]$, firm 1 sends the message $\sigma_{1}(x)$ solving (4) with $\sigma_{1}(-2 \hat{\theta} \gamma)=-2 \hat{\theta} \gamma$ while firm 2 doesn't advertise. If $x \in(-2 \hat{\theta} \gamma, 2 \hat{\theta} \gamma)$, neither firm advertises. If $x \in[2 \hat{\theta} \gamma, 1]$, firm 2 sends the message $\sigma_{2}(x)$ solving (5) with $\sigma_{2}(2 \hat{\theta} \gamma)=2 \hat{\theta} \gamma$ and firm 1 doesn't advertise. If one firm advertises, consumers infer the true quality differential; if neither firm advertises, 
consumers rationally expect a quality differential of zero.

Again the states of the world where firms advertise or are silent are exactly the same as in the pure disclosure framework; see Figure 2.

The proof specifies out-of-equilibrium beliefs that support the equilibrium. The beliefs have the following property: at an out-of-equilibrium information set consumers believe (when possible) that it was reached with the minimum number of deviations from the equilibrium strategies. For instance, the pair of messages $\left(z_{1}, z_{2}\right)$ where $z_{1} \leq-2 \hat{\theta} \gamma$ and $z_{2}<2 \hat{\theta} \gamma$ is not part of the equilibrium path. Should consumers observe such a pair, they believe that firm 1 played its equilibrium strategy while firm 2 got it wrong. Hence, they believe that the true differential is $x$ solving $\sigma_{1}(x)=z_{1}$. A similar refinement, which we call the minimality condition, has been used by Bagwell and Ramey (1991), Schultz (1999), and Emons and Fluet (2009).

For the quadratic cost function $c\left(z_{i}, x\right)=\gamma+.5\left(z_{i}-x\right)^{2}, z_{1}$ and $z_{2}$ satisfy

$$
\begin{aligned}
& z_{1}+\left[1-e^{2 \hat{\theta}\left(z_{1}+2 \hat{\theta} \gamma\right)}\right] / 2 \hat{\theta}=x \text { with } z_{1} \leq x \leq-2 \hat{\theta} \gamma \\
& z_{2}-\left[1-e^{-2 \hat{\theta}\left(z_{2}-2 \hat{\theta} \gamma\right)}\right] / 2 \hat{\theta}=x \text { with } z_{2} \geq x \geq 2 \hat{\theta} \gamma
\end{aligned}
$$

These strategies are illustrated in Figure 5. The quadratic cost function satisfies the no-jamming conditions if $\gamma \geq 1 /(4 \hat{\theta})^{2}$.

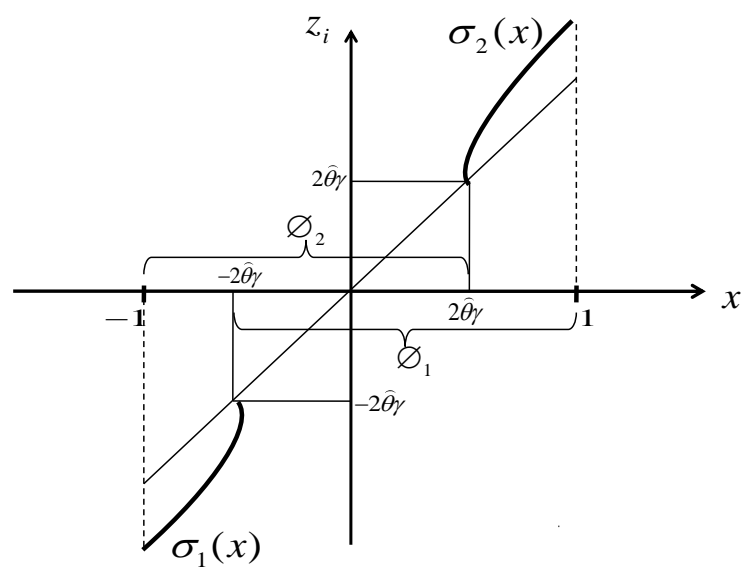

Figure 5: The signalling strategies with quadratic costs 


\subsection{Welfare}

We now compare the equilibria described in the Propositions 3 and 4 . The welfare comparison is the same as in the disclosure set-up in the areas $a, d$ and $f$; see Figure 3. The comparison is ambiguous in region $b$ for the same reasons as under pure disclosure.

Consider the areas $c_{2}$ and $e$ in the top half of the figure. Along the lower boundary of these areas, the cost of the comparative message is $\gamma$. The cost of a non-comparative ad is at least as large because messages incorporate boasting except when $q_{2}=4 \hat{\theta} \gamma$; moreover costs are duplicated in region $e$. Thus, along the lower boundary of $c_{1}$ and $e$ comparative advertising performs better, either because consumers are better informed or the overall advertising expenditure is smaller. By continuity, there is, therefore, a region in $c_{1}$ and $e$ where comparative advertising does better. Without additional assumptions, however, one cannot be sure that comparative advertising does better over the whole of the areas $c_{1}$ and $e$. The reason is that the comparison of advertising costs can go either way. ${ }^{13}$

Finally, consider the area $c_{1}$. At the lower boundary, i.e., for $q_{2}=4 \hat{\theta} \gamma$, a non-comparative ad costs $\gamma$; a comparative ad delivers more information but is typically more expensive. Thus, although comparative advertising must perform better in some part of $c_{1}$, the comparison is generally ambiguous in this area. Overall, in the signalling framework non-comparative advertising does somewhat less well than comparative advertising compared to the disclosure framework.

As a particular case, consider our quadratic cost example. Here we have the following result.

Lemma 3: With quadratic costs $c(y, q)=\gamma+.5(y-q)^{2}$ and $c(z, x)=\gamma+$ $.5(z-x)^{2}$, advertising costs at equilibrium are larger for the non-comparative than for the comparative advertiser in $c_{2}$ and $e$ and lower in $c_{1}$.

\footnotetext{
${ }^{13}$ This is true even when comparative and non- comparative ads involve the same cost function $c(\cdot, \cdot)$.
} 
In $c_{2}$ the non-comparative advertiser has the same fixed cost but a larger variable signalling cost than the comparative advertiser. Moreover, noncomparative advertising provides less information. Thus, information is better and signalling less costly under comparative advertising.

In $e$ the information is the same under both regimes. However, each non-comparative advertiser has a higher signalling cost than the single comparative advertiser: the fixed cost is the same, and variable cost is higher. Furthermore, costs are duplicated under non-comparative advertising.

\section{Concluding Remarks}

The purpose of this paper is to analyze non-comparative and comparative advertising in a framework where firms disclose or signal their quality. To do so, we develop a model that allows for a meaningful analysis of non-price signalling shutting down the channel of price signalling. In the least-cost equilibria of the signalling game firms advertise in exactly the same states as they do in the unique equilibria of the disclosure game. In both frameworks comparative advertising tends to perform better than non-comparative advertising: firms do not advertise at all if the informational content is of little value to consumers; moreover, they never advertise together. By contrast, under non-comparative advertising a firm advertises if its quality level is above a threshold. When both firms have high quality, both advertise and there is a duplication of advertising costs.

We have considered a model where the market is covered so that only the quality differential matters, which obviously makes a strong case for comparative advertising. If, for example, prices are so high that neither loyal nor quality-conscious customers with $\theta$ close to zero buy, marginal consumers do not care about the quality differential; they care only about the quality of their favorite designs. In this case firms will only use non-comparative advertising and allowing for comparative advertising will have no effect. 
Nevertheless, in markets where consumers directly compare products there is scope for comparative advertising to improve the allocation through better information and lower advertising expenditures. Our result that only one firm uses comparative advertising is obviously driven by our one-dimensional quality assumption. As noted in the introduction, the result is at odds with the empirical findings of Anderson et al. (2010 a,b) where all firms used comparative advertising. Arguably, in the analgesics industry there are multiple quality features and firms claim superiority in the dimensions where they perform better. Analyzing comparative advertising with multiple quality features is an interesting topic for future research.

In our analysis of the signalling set-up we focused on separating equilibria. When comparative advertising is allowed, this entailed a two-sender communication game where both firms can potentially send conflicting signals about the same variable. We assumed that signalling costs satisfied a "no-jamming" condition which ensured separation in an equilibrium with the same basic structure as under pure disclosure. Relaxing this condition is another topic for future research. Presumably this will bear on the welfare analysis of comparative versus non-comparative advertising.

\section{Appendix}

Proof of Lemma 2. We complete the argument ruling out pairs of messages of the form $\left(q_{1}^{\prime}, q_{2}\right)$. The following notation different from what we use in the main text turns out to be useful. We denote $i$ 's action by $\left(y_{1}^{i}, y_{2}^{i}\right)$ where $y_{j}^{i} \in\left\{q_{j}, \emptyset_{j}^{i}\right\}$ is the statement about firm $j$ 's quality; $q_{j}$ means that $j$ 's quality is disclosed; $\emptyset_{j}^{i}$ means that $i$ says nothing about $j$ 's quality; $\left(\emptyset_{1}^{i}, \emptyset_{2}^{i}\right)$ means that $i$ does not advertise. A pair of messages is denoted by $\left(\left(y_{1}^{1}, y_{2}^{1}\right),\left(y_{1}^{2}, y_{2}^{2}\right)\right)$. We want to show that a pair of the form $A:=\left(\left(q_{1}^{\prime}, \emptyset_{2}^{1}\right),\left(\emptyset_{1}^{2}, q_{2}\right)\right)$ is never played at equilibrium.

Suppose the contrary. Given that firm 2 advertises when the state is $\left(q_{1}^{\prime}, q_{2}\right)$, by monotonicity it also does at $\left(q_{1}, q_{2}\right)$ for $q_{1}<q_{1}^{\prime}$. From the argument in the text, we know that, for $q_{1}$ sufficiently small, firm 1 will not advertise when firm 2 plays $\left(\emptyset_{1}^{2}, q_{2}\right)$. From the same argument, we also know, however, that no-advertising by firm 1 together with firm 2 playing $\left(\emptyset_{1}^{2}, q_{2}\right)$ is not on the equilibrium path. The only remaining possibility, therefore, is the existence of some critical value $q_{1}^{c} \in\left(0, q_{1}^{\prime}\right)$ 
at which (i) firm 2 switches from non-comparative to comparative advertising and (ii) firm 1 switches from non-comparative advertising to no-advertising.

Consider the state $\left(q_{1}^{c}+\varepsilon, q_{2}\right)$ where $\varepsilon>0$ and small. At that state both firms reveal their own quality. In order for firm 1 not to deviate to no-advertising, consumers, upon observing $B:=\left(\left(\emptyset_{1}^{1}, \emptyset_{2}^{1}\right),\left(\emptyset_{1}^{2}, q_{2}\right)\right)$, must hold a belief $\hat{q}_{1}<q_{1}^{c}$. Consider now the state $\left(q_{1}^{c}-\eta, q_{2}\right)$ where $\eta>0$. Now firm 1 does not advertise, while firm 2 reveals both qualities. If firm 2 deviates to non-comparative advertising, consumers also observe $B$. For $\eta$ small enough, $\hat{q}_{1}<q_{1}^{c}-\eta$, implying that the deviation is strictly profitable for firm 2 .

Proof of Proposition 4. We provide out-of-equilibrium beliefs supporting the equilibrium. It suffices to consider the case where firm 1 plays its prescribed strategy while firm 2 deviates. The argument is similar for deviations by firm 1 .

a) Let $x>-2 \hat{\theta} \gamma$ so that firm 1 plays $\emptyset_{1}$ at equilibrium. The pair of messages observed by consumers is off the equilibrium path if firm 2 sends a a noncomparative message $y_{2}$ or a comparative message $z_{2}<2 \hat{\theta} \gamma$. In either case, from the minimality condition, consumers believe that firm 2 deviated while firm 1 stuck to its equilibrium strategy. Hence, they must hold beliefs consistent with the play of $\emptyset_{1}$ by firm 1 , i.e., they believe that $x$ belongs to $(-2 \hat{\theta} \gamma, 1]$. Any inference $\hat{x} \in(-2 \hat{\theta} \gamma, 2 \hat{\theta} \gamma)$ is sufficient to deter such deviations by firm 2 . The argument is that these deviations are dominated by the play of $\emptyset_{2}$, in which case consumers would infer $\hat{x}=0$; that is, compared to the play of $\emptyset_{2}$, advertising costs at least $\gamma$ and is not worth the cost if consumers believe $\hat{x}<2 \theta \gamma$.

b) Let $x \leq-2 \hat{\theta} \gamma$ so that firm 1 plays $z_{1}=\sigma_{1}(x)$ at equilibrium. The pair of messages observed by consumers is off the equilibrium path if firm 2 sends a a non-comparative message $y_{2}$, a comparative message $z_{2}<2 \hat{\theta} \gamma$ or a comparative message $z_{2} \geq 2 \hat{\theta} \gamma$. In the first two cases, from the minimality condition, consumers believe that firm 2 deviated while firm 1 played its equilibrium strategy. Hence they infer that the true differential is the solution to $z_{1}=\sigma_{1}(x)$. Because advertising costs are incurred without affecting beliefs, the first two deviations are therefore not profitable for firm 2 .

In the third case, consumers observe a pair of messages of the form $\left(z_{1}, z_{2}\right)$ with $z_{1} \leq-2 \hat{\theta} \gamma$ and $z_{2} \geq 2 \hat{\theta} \gamma$. They therefore know that at least one firm has deviated but do not know which. From the minimality condition, they believe that at most one did. Thus, they believe that $x \in\left\{\sigma_{1}^{-1}\left(z_{1}\right), \sigma_{2}^{-1}\left(z_{2}\right)\right\}$. Suppose they believe the two possibilities are equiprobable. Their inference is then

$$
\hat{x}=.5 \sigma_{1}^{-1}\left(z_{1}\right)+.5 \sigma_{2}^{-1}\left(z_{2}\right)=.5 x+.5 x_{2}
$$

where $x=\sigma_{1}^{-1}\left(z_{1}\right)$ because firm 1 is playing its equilibrium strategy and where $x_{2}:=\sigma_{2}^{-1}\left(z_{2}\right)$ is the quality differential that firm 2 is mimicking. Written as a 
function of the latter and of the true state, firm 2's profit is

$$
\pi_{2}\left(x_{2}, x\right)=.5+\left(x+x_{2}\right) / 4 \hat{\theta}-c\left(\sigma_{2}\left(x_{2}\right), x\right) .
$$

The profit from its prescribed equilibrium strategy $\emptyset_{2}$ is

$$
\pi_{2}\left(\emptyset_{2}, x\right)=.5+x / 2 \hat{\theta}
$$

Define

$$
\varphi\left(x_{2}, x\right):=\pi_{2}\left(x_{2}\right)-\pi_{2}\left(\emptyset_{2}\right)=\left(x_{2}-x\right) / 4 \hat{\theta}-c\left(\sigma_{2}\left(x_{2}\right), x\right) .
$$

We show that $\varphi\left(x_{2}, x\right)<0$ for all $x \leq-2 \hat{\theta} \gamma$ and $x_{2} \geq 2 \hat{\theta} \gamma$.

First

$$
\begin{aligned}
\varphi_{x_{2}}\left(x_{2}, x\right) & \left.=1 / 4 \hat{\theta}-c_{z}\left(\sigma_{2}\left(x_{2}\right), x\right) \sigma_{2}^{\prime}\left(x_{2}\right)\right) \\
& =1 / 4 \hat{\theta}-c_{z}\left(\sigma_{2}\left(x_{2}\right), x\right) / 2 \hat{\theta} c_{z}\left(\sigma_{2}\left(x_{2}\right), x_{2}\right)<0
\end{aligned}
$$

where we substituted for $\sigma_{2}^{\prime}\left(x_{2}\right)$ from condition (5). The inequality follows from the single-crossing property and the fact that $x_{2}>x$. Thus $\varphi\left(x_{2}, x\right)$ is maximized at $x_{2}=2 \hat{\theta} \gamma$. Now

$$
\varphi(2 \hat{\theta} \gamma, x)=-x / 4 \hat{\theta}+\gamma / 2-c(2 \hat{\theta} \gamma, x)
$$

In particular,

$$
\varphi(2 \hat{\theta} \gamma,-2 \hat{\theta} \gamma)=\gamma-c(2 \hat{\theta} \gamma,-2 \hat{\theta} \gamma)<0
$$

Next we show that $\varphi(2 \hat{\theta} \gamma, x)$ is maximized at $x=-2 \hat{\theta} \gamma$. Differentiating with respect to $x$,

$$
\varphi_{x}(2 \hat{\theta} \gamma, x)=-1 / 4 \hat{\theta}-c_{x}(2 \hat{\theta} \gamma, x) \geq 0 \text { for } x \leq-2 \hat{\theta} \gamma
$$

The inequality follows from the no-jamming condition. Hence

$$
\varphi\left(x_{2}, x\right) \leq \varphi(2 \hat{\theta} \gamma, x) \leq \varphi(2 \hat{\theta} \gamma,-2 \hat{\theta} \gamma)<0
$$

for all $x \leq-2 \hat{\theta} \gamma$ and $x_{2} \geq 2 \hat{\theta} \gamma$.

Proof of Lemma 2. Consider firm 2. Let $\hat{s}\left(q_{2}\right):=s\left(q_{2}\right)-q_{2}$. Substituting in (3) yields

$$
\hat{s}\left(q_{2}\right)=\left[1-e^{-2 \hat{\theta}\left(\hat{s}\left(q_{2}\right)+q_{2}-4 \hat{\theta} \gamma\right)}\right] / 2 \hat{\theta}, \quad \text { for } q_{2} \geq 4 \hat{\theta} \gamma
$$


Likewise, let $\hat{\sigma}_{2}(x):=\sigma_{2}(x)-x$. Substituting in (5),

$$
\hat{\sigma}_{2}(x)=\left[1-e^{-2 \hat{\theta}\left(\hat{\sigma}_{2}(x)+x-2 \hat{\theta} \gamma\right)}\right] / 2 \hat{\theta}, \quad \text { for } x \geq 2 \hat{\theta} \gamma .
$$

Let $t$ and $t^{\prime}$ be such that $\hat{s}\left(t^{\prime}\right)=\hat{\sigma}_{2}(t)$. Thus

$$
\left[1-e^{-2 \hat{\theta}\left(\hat{s}\left(t^{\prime}\right)+t^{\prime}-4 \hat{\theta} \gamma\right)}\right] / 2 \hat{\theta}=\left[1-e^{-2 \hat{\theta}\left(\hat{\sigma}_{2}(t)+t-2 \hat{\theta} \gamma\right)}\right] / 2 \hat{\theta}
$$

which yields

$$
t^{\prime}=t+2 \hat{\theta} \gamma
$$

Since $\hat{s}(\cdot)$ and $\hat{\sigma}_{2}(\cdot)$ are increasing functions, it follows that for $t^{\prime}>t+2 \hat{\theta} \gamma$, $\hat{s}\left(t^{\prime}\right)>\hat{\sigma}_{2}(t)$. Conversely, $t^{\prime}<t+2 \hat{\theta} \gamma$ implies $\hat{s}\left(t^{\prime}\right)<\hat{\sigma}_{2}(t)$.

With the quadratic cost function, advertising costs are larger for a non-comparative than for a comparative ad when $\hat{s}\left(q_{2}\right) \geq \hat{\sigma}_{2}\left(q_{2}-q_{1}\right)$ and are smaller otherwise. In the areas $c_{2}$ and $e$ of Figure $3, q_{1} \geq 2 \hat{\theta} \gamma$ so that $q_{2} \geq q_{2}-q_{1}+2 \hat{\theta} \gamma$. From the preceding result, it follows that $\hat{s}\left(q_{2}\right) \geq \hat{\sigma}_{2}\left(q_{2}-q_{1}\right)$. In the area $c_{1}, q_{1}<2 \hat{\theta} \gamma$ so that $q_{2}<q_{2}-q_{1}+2 \hat{\theta} \gamma$. Hence, $\hat{s}\left(q_{2}\right)<\hat{\sigma}_{2}\left(q_{2}-q_{1}\right)$.

\section{References}

Anderson, S., Renault, R., 2009. Comparative Advertising: Disclosing Horizontal Match Information, Rand Journal of Economics 40, 558-581.

Anderson, S., Ciliberto, F., Liaukonyte, J., 2010. Getting into Your Head(Ache): The Information Content of Advertising in the Over-the-Counter Analgesics Industry, ssrn.com/abstract $=1647825$

Anderson, S., Ciliberto, F., Liaukonyte, J., Renault, R., 2010. PushMe Pull-You: Advertising in the OTC Analgesics Industry, faculty.cit.cornell.edu/ j12545/papers/IIOC2010.pdf

BAgwell, K., 2007. The Economic Analysis of Advertising, in M. Amstrong and R. Porter, Eds., Handbook of Industrial Organization, 3, North Holland, Amsterdam, 1701-1829.

Bagwell, K., Ramey, G., 1991. Oligopoly Limit Pricing, Rand Journal of Economics 22, 155-72.

Barigozzi, F., Peitz, M., 2006. Comparative Advertising and Competition Policy, in: J. P. Choi (ed.), Recent Trends in Antitrust: Theory and Evidence, MIT Press, Cambridge MA, 215-263.

Barigozzi, F., Garella, P., Peitz, M., 2009. With a little Help from my Enemy: Comparative Advertising as a Signal of Quality, Journal of Economics and Management Strategy 18, 1071-1094. 
Daughety, A., Reinganum, J., 2007. Competition and Confidentiality: Signalling Quality in a Duopoly when there is Universal Private Information, Games and Economic Behavior 58, 94-120.

Daughety, A., Reinganum, J., 2008. Communicating Quality: A Unified Model of Disclosure and Signalling, Rand Journal of Economics 39, 973-989.

Emons, W., Fluet, C., 2009. Accuracy versus Falsification Costs: The Optimal Amount of Evidence under different Procedures, Journal of Law, Economics, and Organization 25, 134-156.

Fluet, C., Garella, P., 2002. Advertising and Prices as Signals of Quality in a Regime of Price Rivalry, International Journal of Industrial Organization 20, 907-930.

Grewal, D., Kavanoor, S., Fern, E., Costley, C., Barnes, J., 1997. Comparative versus Non-comparative Advertising: A Meta-Analysis, Journal of Marketing 61, 1-15.

Hertzendorf, M., Overgaard, P., 2001. Price Competition and Advertising Signals: signalling by Competing Senders, Journal of Economics and Management Strategy 10, 621-662.

Kartik, N., 2009. Strategic Communication with Lying Costs, Review of Economic Studies 76, 1359-95.

KIM, J.-Y., 2003. Signal Jamming in Games with Multiple Senders, Contributions to Theoretical Economics 3, Article 7.

Levin, D., Peck, J., Ye, L., 2009. Quality Disclosure and Competition, Journal of Industrial Economics 57, 167-196.

Mailath, G., 1987. Incentive Compatibility in Signaling Games with a Continuum of Types, Econometrica 55, 1349-1365.

Milgrom, P., Roberts, J., 1986a. Prices and Advertising Signals of Product Quality, Journal of Political Economy 94, 796-821.

Milgrom, P., Roberts, J., 1986b. Relying on the Information of Interested Parties, Rand Journal of Economics 17, 18-32.

Schultz, C., 1999. Limit Pricing when Incumbents have Conflicting Interests, International Journal of Industrial Organization 17, 801-825. 